\title{
Freud y Ferenczi: algunas consideraciones sobre la creación de la primera cátedra de psicoanálisis en la Universidad*1
}

\section{Freud and Ferenzi: some thoughts on the creation of the first chair of psychoanalysis at the University}

Francisco Pizarro Obaid*2

\begin{abstract}
El presente artículo expone y analiza las reflexiones que sostuvieron Freud y Ferenczi durante el proceso de creación de la primera cátedra de psicoanálisis en la Universidad de Budapest. A partir de estos elementos, se destacará que no fueron sólo diferencias teóricas o profesionales las que marcaron la relación conflictiva entre el psicoanálisis y la Universidad, sino que en ese proceso influyó de manera decisiva la política institucional que fundamentó la constitución del movimiento psicoanalítico.
\end{abstract}

Palabras claves: Freud, Ferenczi, Universidad, Movimiento

Psicoanalítico

*1 Articulo basado en la presentación oral "Algunas reflexiones de Freud y Ferenczi sobre las relaciones del Psicoanálisis y la Universidad"; XXV Congreso Latinoamericano sobre el pensamiento de D. W.Winnicott: Clínica de la neurosis y la psicosis. Variedades de la transferencia; 11 Noviembre de 2016; Universidad Diego Portales, Santiago, Chile.

*2 Universidad Diego Portales (Santiago, Chile) 


\section{Introducción}

Desde comienzos de este milenio los posibles vínculos entre el psicoanálisis y la universidad han sido asunto de un activo debate (Garza-Guerrero, 2002a; Kernberg, 2011). El estudio del problema ha involucrado aspectos institucionales (Garza-Guerrero, 2002b; Kernberg, 2006a; Roussillon, 2010), académicos (Eifermann, 1993; Blass, 2001; Laplanche, 2004; Garza-Guerrero, 2004; Kernberg, 2007), así como también, controversias sobre la formación del analista, el estatuto de la investigación (Kernberg, 2006b) y las posibles relaciones con el ámbito universitario (Wallerstein, 2009; 2011; Kernberg, 2011; Long, Eagle, \& Stevens, G. (2015). Si bien la discusión está lejos de ser concluyente, entre sus distintos capítulos las palabras crisis, conflicto, peligro, amenaza, complejidad, se vuelven recurrentes para dar cuenta del estado del problema. Pero, como muestra la historia del psicoanálisis, esta dinámica no responde a la contingencia o a una polémica asilada, sino más bien a una constante en el desarrollo de la institucionalidad psicoanalítica (Roudinesco \& Plon, 1997; Rodrigué, 1996; Breger, 2001; Gay, 2006).

Una breve revisión de algunos antecedentes históricos referidos a la creación de la primera cátedra de psicoanálisis en la Universidad de Budapest, permitirá ilustrar, por una parte, que la relación entre estos dos campos - Psicoanálisis/Universidad — fue siempre conflictiva y, por la otra, que en el debate sobre las posibles articulaciones entre la Institución psicoanalítica y la Academia, no sólo intervinieron diferencias teóricas o profesionales, sino que, desde el inicio, la dimensión política - en su sentido general y en su particularidad institucional - fue parte del asunto. 
En esta perspectiva, el proyecto universitario llevado a cabo por Ferenczi, en estrecha colaboración con Freud, fue solidario con el ideal freudiano de crear un movimiento psicoanalítico, política que permitió un crecimiento y una expansión institucional del psicoanálisis, pero, al mismo tiempo, fue una de las variables que marcó su distanciamiento del campo académico.

\section{Freud y Ferenczi frente a las posibles relaciones entre el Psicoanálisis y la Universidad}

A lo largo de su vínculo de amistad y trabajo, Freud y Ferenczi sostuvieron diálogos sobre materias particularmente polémicas. Además de las notables reflexiones sobre teoría y técnica psicoanalítica, alguna vez pensaron investigar las relaciones entre la neurosis y la filogenia (Ferenczi, 1915/1970; Freud \& Ferenczi, 1914-1919/1992); explorar los vínculos entre la neurosis y la teoría de Lamarck (Freud \& Ferenczi, 1914-1919/1992) o, incluso, se vieron seducidos por el estudio del ocultismo y la telepatía (Maureau, 1983). Sin ese carácter altamente especulativo, también compartieron su preocupación por las posibles relaciones entre el psicoanálisis y la universidad, un tópico, sin lugar a dudas, polémico.

Freud (1919/1996b) expuso por primera vez, "oficialmente", sus reflexiones sobre una eventual inserción del psicoanálisis en la Universidad mediante un artículo publicado en la revista médica Gyógyászat. Se trataba de un texto en húngaro - probablemente traducido por Ferenczi —, donde el Profesor exponía su posición frente al problema, tomando como referencia el título del escrito: “¿Debe enseñarse el psicoanálisis en la universidad?”. ${ }^{1} \mathrm{Su}$ respuesta explicitaba de inmediato que la relación entre las dos instituciones no era, de ningún modo, armónica. Destacaba que las asociaciones

${ }^{1}$ La opción de establecer un título con forma de pregunta es significativo. Si obviamos su correspondencia - donde, por ejemplo, encontramos el Manuscrito E (1894) que se organiza bajo el título ¿Cómo se genera la angustia? o el intercambio epistolar con Einstein donde encontramos el título ¿Por qué la guerra? (Freud, 1933[1932]/1996) — sólo dos títulos son formulados como pregunta y están íntimamente enlazados: ¿Debe enseñarse el psicoanálisis en la universidad? (1919[1918]/1996g) y ¿Pueden los legos ejercer el análisis? Diálogos con un juez imparcial (1926/1996f). 


\section{HISTÓRIA DA PSICANÁLISE}

psicoanalíticas habían surgido, justamente, a partir de la exclusión del psicoanálisis por parte de la universidad (Freud, 1919[1918]/1996c, p. 169), para luego advertir que las sociedades tendrían utilidad mientras se mantuviera ese rechazo. Si bien, un posible ingreso del psicoanálisis a la universidad podría ser considerado una "satisfacción moral" (p. 169), su reconocimiento plantearía el desafío de pensar, a su vez, cuáles serían las condiciones de su enseñanza en ese contexto institucional.

Al analizar el problema académico, Freud (1919[1918]/1996c) criticó fuertemente el plan de estudio que la universidad definía para la formación de especialistas en el tratamiento de las enfermedades nerviosas. Denunció la escasa importancia otorgada por la medicina al factor psíquico (privilegio de la anatomía, la física y la química), así como el carácter eminentemente descriptivo de la aproximación psiquiátrica al campo psicopatológico (vinculación de los signos con una etiología orgánica y un correlato anatomopatológico). A su juicio, estas opciones teóricas y metodológicas habían conducido a la instauración de cursos de psicología médica basados en la "psicología escolástica" o en la "psicología experimental" (p. 170). Ante estas deficiencias, el psicoanálisis sería, según Freud (1919[1918]/1996c), la teoría más idónea para el conocimiento cabal de la psicología y la gran vía explicativa para los fenómenos psicopatológicos; dada su potencia, el psicoanálisis debería ser considerado "[...] el término y culminación de toda psicoterapia" (p. 170).

Pero la teoría psicoanalítica no podria restringirse a la disciplina psicológica o médica, ni ser reducida a un técnica de intervención psicoterapéutica o a un método exclusivamente clínico; para Freud (1919[1918]/1996c) el Psicoanálisis debía ser considerado, igualmente, un nuevo método de investigación que permitiría explorar problemas "artísticos, filosóficos o religiosos, suministrando en tal sentido múltiples enfoques nuevos y revelaciones de importancia para la historia de la literatura, la mitología, la historia de las culturas y la filosofía de las religiones" (p. 171). De este modo, concluía, "la universidad únicamente puede beneficiarse con la asimilación del psicoanálisis en sus planes de estudio" (p. 171).

Bajo estas puntualizaciones, la universidad debía ser definida como "un lugar donde se imparte el conocimiento por encima de todas las diferencias de religiones y de nacionalidades, donde se investiga, para mostrar a la humanidad hasta qué punto comprenden el mundo que los rodea y hasta qué punto pueden controlarlo" (Freud, 1925/1996e, p. 302). Por lo tanto, la condición básica era para Freud (1919/1996b), saber si la universidad estaría “(...) dispuesta a reconocer al psicoanálisis alguna importancia en la 
formación del médico y del hombre de ciencia. De ser así, tendrá que resolver la manera de incluirlo en el conjunto de su enseñanza" (p. 169). El escenario no era el más propicio, ya que las transformaciones sufridas tras la primera guerra, incidirían inevitablemente en las pretensiones freudianas.

En un contexto de restructuración geopolítica, marcado por el ocaso del imperio Austro-Húngaro, Ferenczi tuvo un protagonismo crucial en el devenir del Psicoanálisis, al poner en práctica los criterios freudianos expuestos en el artículo de Gyógyászat (Freud, 1919/1996b) y al sortear una serie de gestiones políticas que lo condujeron, finalmente, a detentar el cargo de Professor de psicoanálisis en la universidad de Budapest, dando lugar a la creación de la primera cátedra de Psicoanálisis en la Universidad.

\section{Ferenczi y el periplo por la universidad}

Las primeras relaciones curriculares entre el psicoanálisis y la univer$\operatorname{sidad}^{2}$ estuvieron marcadas por la serie de acontecimientos políticos derivados de la Primera guerra mundial, eventos que produjeron, entre sus diversas consecuencias, la disolución de la monarquía austro-húngara.

El 17 de octubre Tisza notificaba al parlamento húngaro que la guerra había sido, finalmente, perdida y advertía sobre el caótico retorno de los soldados desde el frente. Ferenczi, por su parte, confesaba a Freud, con tono resuelto: “(...) acabo de terminar mi triste adiós a la Hungría de antes” (Freud \& Ferenczi, 1992, p. 334). La respuesta freudiana no se haría esperar: "Retire a tiempo su libido de la patria y póngala a resguardo en el psicoanálisis, si no, usted va a sentir, necesariamente, mal" (p. 336).

Pese a los apremios de la vida, las restricciones en la comunicación y las dificultades para mantener la cohesión del grupo psicoanalítico en el contexto de catástrofe, en octubre Ferenczi relataba a Freud que algunos estudiantes de medicina de la universidad de Budapest le habían solicitado la realización de conferencias sobre psicoanálisis: "He aceptado a condición de que dispongan de un local conveniente. Instantáneamente, un movimiento se ha desatado!

${ }^{2}$ Se deben descontar las actividades de extensión (Conferencias en la Clark University, 1909) o los cursos dictados por Freud en la Universidad de Viena, en su calidad de Docente (Privat Dozent; Profesor Extraordinarius). 


\section{HISTÓRIA DA PSICANÁLISE}

180 firmantes quieren dirigir al rector de la universidad una petición para que me den la posibilidad de enseñar" (Freud \& Ferenczi, p. 335).

Asimismo, los estudiantes expresarían, más tarde, su deseo de fundar una asociación psicoanalítica de estudiantes de medicina, iniciativa que Ferenczi estaba dispuesto a apoyar.

Hacia fines de Diciembre de 1918, la viabilidad de lo que Ferenczi (denominaba "psicoanálisis académico" (Freud \& Ferenczi, 1992, p. 349) encontraba una férrea oposición, tanto en el ámbito universitario, así como en la esfera gubernamental. El ingreso a la universidad sólo sería posible, según Ferenczi, si el gobierno "(...) hace una concesión al psicoanálisis (así como otorga el derecho a voto) (...)" (Freud \& Ferenczi, 1992, p. 349).

Pese a las sombrías circunstancias por las cuales atravesaba Europa, Ferenczi reportaba, con entusiasmo, a Freud: “(...) algunos adeptos al Psicoanálisis han reunido firmas para una petición a la universidad (cerca de 1.000 hasta ahora); piden que les demos la posibilidad de estudiar Psicoanálisis" (Freud \& Ferenczi, 1992, p. 345). Luego agregaba, con fervor, "La patria del Psicoanálisis es, pese a todo, Budapest y no Viena; usted debería instalarse aquí" (p. 345).

A comienzos de 1919, la inserción del psicoanálisis comenzó a tomar forma y las gestiones políticas se intensificaron. Ferenczi precisaba a Freud el alcance que habían tenido las demandas en favor del psicoanálisis:

(...) el nuevo Ministro de educación está bien dispuesto hacia la causa, así como también el Comisario del gobierno, recientemente nombrado para la universidad. Nuestra Asociación ha dirigido un memorándum al ministerio; los estudiantes han enviado, a su vez, una petición en el mismo sentido. El asunto puede decidirse en algunas semanas. (Freud \& Ferenczi, 1992, p. 367)

Pese a la suspicacia expresada por el nuevo orden político y administrativo, el 25 de abril de 1919 Ferenczi fue finalmente nombrado Profesor de la cátedra de psicoanálisis y director de la clínica psiquiátrica asociada a la universidad, bajo la República de los Consejos. ${ }^{3}$ En junio, Freud (1919/1996b) escribía Jones: "Hasta aquí la 'Rateregierung' (el gobierno comunista) se ha

${ }^{3}$ Según Jones (1957) "Rado tenía cierta influencia con los dueños de la situación y fue él quien lo había conseguido. Róheim había sido designado profesor de antropología un par de semanas antes. Ferenczi habría de pagar bien cara esta incauta aceptación de tal honor. Luego que los rumanos penetraron en Budapest, en el mes de agosto, el régimen reaccionario que se implantó fue violentamente antisemita y por largo tiempo Ferenczi tuvo temor de mostrarse en las calles de la ciudad" (p.16). 
conducido muy gentilmente hacia nuestra ciencia. Ferenczi se ha convertido en el primer profesor oficial de psicoanálisis en la universidad (Professor), un éxito que no habíamos soñado nunca!"' (p. 419).

Pero ¿Cuál era el programa que Ferenczi quería impartir en la universidad? Un programa que se apoyaba, plenamente, en el artículo freudiano sobre las relaciones entre psicoanálisis y universidad, publicado en Gyógyászat. El acuerdo con los argumentos expresados por Freud (1919/1996b), a favor y en contra de la universidad, era pleno y Ferenczi sólo proponía introducir una breve frase, que rezaba: "Es necesario consagrar una sección psiquiátrica a la investigación psicoanalítica sobre las psicosis" (Freud \& Ferenczi, 1992, p. 381).

El plan de trabajo se organizaba en un curso sobre la "Psicología psicoanalítica para médicos" (Freud \& Ferenczi, 1992, p. 397), que constaba de tres horas por semana. En la primera parte, se expondría de manera general la posición del psicoanálisis en relación a otras ciencias: ciencias de la naturaleza, filosofía, psicología, etc. En la segunda y tercera parte, la tarea sería discutir la influencia de lo psíquico en lo físico. Luego vendrían las exposiciones sobre la hipnosis, la sugestión y otras materias. Finalmente, sería el turno del psicoanálisis, tal como Freud (1919[1918]/1996c) lo había esbozado.

Todo parecía auspicioso; definitivamente el psicoanálisis ingresaría a la universidad. Sin embargo, la convulsionada restructuración política desató una seguidilla de hechos — caída de la República de los Consejos (pro soviético) por un golpe de Estado; toma del poder por un grupo derechista y antisemita (Terror Blanco) - que marcaron, en un par de meses, la salida de Ferenczi, tanto de la universidad, así como de la Real Academia Médica de Budapest (Mészáros, 2012). De manera súbita, Ferenczi se vio excluido del campo académico y, dada la precaria situación política de Hungría, en octubre cedió a Ernest Jones la presidencia de la Asociación Psicoanalítica, antes del término oficial de su mandato.

En 1920, Karl Abraham (1969) calificaba la primera incursión oficial de un psicoanalista en la universidad como "La efímera cátedra de Ferenczi en Budapest" (p. 306).

\section{EI movimiento psicoanalítico, la política educativa y el ejercicio profesional}

Después de la breve incursión de Ferenczi en la universidad de Budapest, las actividades formativas e investigativas se concentraron, casi exclusivamente, en las Asociaciones e Institutos psicoanalíticos. 


\section{HISTÓRIA DA PSICANÁLISE}

No obstante, algunos años después, en un contexto muy particular e inesperado, Freud (1926/1996f) retomó la discusión sobre las relaciones entre el psicoanálisis y la universidad. Theodor Reik - miembro no médico de la Sociedad Psicoanalítica de Viena - había sido acusado de violar una antigua ley vigente en Viena contra el "curanderismo", causa que abrió, nuevamente, las preguntas acerca de la formación del analista y los requisitos legales para el ejercicio profesional. En un "diálogo con un juez imparcial", Freud (1926/1996f) se preguntaba “¿Cómo y dónde se aprende lo necesario para el ejercicio del análisis?” (p. 213); “Es el ejercicio del psicoanálisis una materia que deba estar sometida a la intervención de la autoridad, o es más adecuado dejarlo librado a su desarrollo natural?” (p. 220-221); “¿Cuál es la formación más apropiada para los analistas?” (p. 236).

Tras denunciar que la medicina se había arrogado históricamente el derecho y la exclusividad en el tratamiento de las enfermedades nerviosas, y bajo ese sesgo había atacado al psicoanálisis, Freud (1926/1996f) ofreció una sentencia aún más categórica que la expuesta en 1919, sosteniendo que la formación que la universidad ofrece al médico es “(...) casi la contraria de la que le haría falta como preparación para el psicoanálisis. Le han orientado la atención hacia hechos químicos, físicos, anatómicos, susceptibles de comprobación objetiva, de cuya correcta apreciación y adecuada modificación depende el éxito de la acción médica" (p. 216).

Mediante afirmaciones vehementes contra el orden académico y profesional vigente, la postura freudiana no sólo puso en tensión las relaciones entre la medicina y el psicoanálisis, sino que, al mismo tiempo, cuestionó los posibles vínculos entre el psicoanálisis y la institucionalidad académica consagrada al conocimiento de las enfermedades nerviosas. En consecuencia, Freud (1926/1996f) afirmaba:

El plan de estudios para el analista está todavía por crearse; debe abarcar tanto temas de ciencias del espíritu - psicológicos, de historia de la cultura, sociológicos - como anatómicos, biológicos y de historia evolutiva [...] Es fácil objetar a esa propuesta que no existen tales escuelas superiores de análisis, y que son un reclamo ideal. Muy bien; un ideal, pero uno que puede y debe ser realizado. Y nuestros institutos didácticos, a pesar de su juvenil insuficiencia, ya son el comienzo de esa realización. (p. 236)

De este modo, Freud (1926/1996f) concluía su evaluación del problema y se inclinaba por favorecer el desarrollo del psicoanálisis en las coordenadas de su propia institucionalidad: asociaciones regionales; institutos; asociación internacional. Más allá de las evidentes diferencias de tales agrupaciones, 
todas portaban el sello del proyecto freudiano de constituir un movimiento psicoanalítico (Freud, 1914/1996a), política organizativa que tuvo amplias repercusiones en las relaciones que el psicoanálisis estableció con la universidad, desde la primera experiencia que Ferenczi desarrolló en Budapest y que recorren la historia hasta nuestros días.

\section{El antagonismo entre el movimientos psicoanalítico y la universidad}

Si para Freud (1914/1996a; 1925/1996e) el psicoanálisis constituía un movimiento, ¿qué consecuencias se derivan de una lógica institucional de esta índole y cuáles podrían ser sus efectos en la formación de sus miembros? ¿Qué tipo de conflicto es aquel que participa, inexorablemente, en las posibles relaciones entre el psicoanálisis y la universidad? Más allá de los debates epistemológicos, teóricos o técnicos, que podrían tensionar las relaciones entre estos dos dominios, la lógica involucrada en la construcción del movimiento psicoanalítico, resultó ser un factor de alta influencia y un promotor de la ambivalencia en las relaciones que el psicoanálisis estableció con la universidad.

Para Tourraine (1995) los principios estructurantes de un movimiento social son la identidad, la oposición y la totalidad. El movimiento se constituye como un juego de fuerzas, donde el actor crea una definición de sí mismo, la cual afecta, tanto su propia conducta, así como las interacciones que mantiene con la sociedad; la identidad opera como un elemento diferenciador en medio del conflicto, instituyendo, por una parte, un adversario y, por otra, una conciencia entre los actores presentes. De este modo, la totalidad puede ser considerada como un sistema de acción histórica donde los adversarios se disputan el dominio (Tourraine, 1995). Un claro ejemplo de esta configuración fue la creación de un Comité secreto, grupo que Jones (1912/1998) imaginaba como "(...) un pequeño organismo unido, organizado a la manera de los paladines de Carlomagno, para defender el reino y la política de su maestro" (p. 201). Aunque advertía que esta idea había sido el producto de su "romantisismo" (p. 201), Freud replicaba que la idea había sido suya en los tiempos en que imaginaba un grupo cohesionado por Jung.

La actividad del Comité fue claramente política y fijó los lineamientos institucionales del movimiento psicoanalítico (Grosskurth, 1991; Gellner, 1993). Para Freud, se trataba de un grupo selecto que debería: "velar por el desarrollo ulterior del Psicoanálisis y defender la causa contra las personalidades y los accidentes, cuando yo ya no esté" (Freud \& Jones, 1998, p. 199). 


\section{HISTÓRIA DA PSICANÁLISE}

Las relaciones con la Universidad portaron el mismo sello que Freud (1914/1996a) imprimió a su versión de la historia del movimiento psicoanalítico. Al identificar los orígenes, al describir los escollos o al sintetizar los hitos que marcaron el surgimiento y la expansión del psicoanálisis, la historia freudiana tomó la forma de una epopeya, en la que su protagonista - un hombre solitario, un descubridor genial - batalló, de manera desigual con la ciencia de su época para, finalmente, alzarse como vencedor.

Para dar cuenta de aquella hazaña y con el fin de graficar los desafíos que tuvo que sortear, la memoria freudiana recurrió a expresiones tales como aislamiento, incomprensión, penurias, adquiriendo, así, un tono, eminentemente, biográfico; pero, al mismo tiempo, con el afán de caracterizar su proyecto intelectual, el propio Freud (1914/1996a; 1925/1996e) inscribió términos que tuvieron vastos alcances políticos y sociales, entre los que con insistencia se contarían expresiones tales como: movimiento, maestro, discípulos y doctrina. Una y otra vez las 'historias oficiales' rendirían tributo a la historia escrita por Freud, al invocar los mismos principios y al consignar los mismos hitos establecidos por el Maestro. Bajo estas consideraciones, no es de extrañar que las relaciones entre el psicoanálisis y la universidad hayan estado marcadas por la ambivalencia y rivalidad.

El progresivo distanciamiento del ámbito académico y el fortalecimiento de una actividad societal doctrinaria condujeron a un estado de cosas que el debate contemporáneo diagnosticó en severos términos, al sostener, por ejemplo, que la disciplina psicoanalítica "[...] se originó, evolucionó y aún está enmarcada dentro de una red congregacional que une y fusiona, en forma inextricable y contradictoria, las prerrogativas y funciones que imponen un "movimiento" y una "causa" (cuasi religiosa, ideológica, sociopolítica), con las inherentes a las ciencias y profesiones académicas" (Garza-Guerrero, 2002b, p. 1407).

Si bien aún existen experiencias institucionales donde el psicoanálisis se inserta en la universidad - Departamento de Psicoanálisis de la Universidad de Paris 8; Escuela Doctoral en Paris 7; Laboratorios de investigación en diversas Universidades de Brasil; incluso, una Universidad Psicoanalítica en Berlin - el problema no se resuelve por la conquista institucional ¿Debe enseñarse el psicoanálisis en la universidad? Sin lugar a dudas, pero para ello es necesario que los psicoanalistas se prevengan de las trampas identitarias que recorren la historia del movimiento psicoanalítico y estén dispuestos a enfrentar decididamente las exigencias que impone al quehacer analítico, el desafío de producir, y no sólo transmitir conocimiento; el desafío de inventar y no sólo repetir lo ya conocido. 


\section{Referencias}

Abraham, K., \& Freud, S. (1969). Correspondance (1907-1925). Paris, France: Gallimard.

Blass, R. B. (2001). The Teaching of the Oedipus Complex: On Making Freud Meaningful to University Studens by Unveiling his Essential Ideas on the Human Condition. Int. J. Psycho-Anal, 82, 1105-1121.

Breger, L. (2001). Freud: Darkness in the midst of vision. New York, NY: Wiley.

Eifermann, R. R. (1993). Teaching and Learning in an Analytic Mode - A Model for

Studying Psychoanalysis at University. Int. J. Psycho-Anal., 74, 1005-1015.

Ferenczi, S. (1970). L'ère glaciaire des périls. In Psychanalyse 2, Euvres Complètes

Tome II: 1913-1919. Paris, France: Payot. (Trabajo original publicado en 1915).

Freud, S. (1996). Contribución a la historia del movimiento psicoanalítico. In Obras Completas Sigmund Freud (Vol. 14, Strachey, J. [Ed.], Etcheverry, J. L. [Trad.]). Buenos Aires, Argentina: Amorrortu. (Trabajo original publicado en 1914).

Freud, S. (1996). Kell-e az egyetemen a psychoanalysist tanitani? Gyógyászat, 59(13), 192. (Trabajo original publicado en 1919).

Freud, S. (1996). ¿Debe enseñarse el psicoanálisis en la universidad? In Obras Completas Sigmund Freud (Vol. 18, Strachey, J. [Ed.], Etcheverry, J. L. [Trad.]), Buenos Aires, Argentina: Amorrortu. (Trabajo original publicado en 1919[1918]).

Freud, S. (1996). Mensaje en la inauguración de la universidad Hebrea. In Obras Completas Sigmund Freud (Vol. 20, Strachey, J. [Ed.], Etcheverry, J. L. [Trad.]), Buenos Aires, Argentina: Amorrortu. (Trabajo original publicado en 1925).

Freud, S. (1996). Presentación autobiográfica. In Obras Completas Sigmund Freud (Vol. 20, Strachey, J. [Ed.], Etcheverry, J. L. [Trad.]), Buenos Aires, Argentina: Amorrortu. (Trabajo original publicado en 1925).

Freud, S. (1996). ¿Pueden los legos ejercer el análisis? Diálogos con un juez imparcial. In: Obras Completas Sigmund Freud (Vol. 20, Strachey, J. [Ed.], Etcheverry, J. L. [Trad.]), Buenos Aires, Argentina, Argentina: Amorrortu. (Trabajo original publicado en 1926).

Freud, S. (1996). ¿Por qué la guerra? (Einstein y Freud). In Obras Completas Sigmund Freud (Vol. 22, Strachey, J. [Ed.], Etcheverry, J. L. [Trad.]), Buenos Aires, Argentina: Amorrortu. (Trabajo original publicado en 1933[1932]).

Freud, S., \& Ferenczi, S. (1992). Correspondance (1914- 1919). Paris, France: Calman-Lévy. (Trabajo original publicado en 1914-1919).

Freud, S., \& Jones, E. (1998). Sigmund Freud-Ernest Jones. Correspondance complète (1908-1939). (P. Dauzat, Trad.). Paris, France: PUF.

Garza-Guerrero, C. (2002a). 'The Crisis In Psychoanalysis': What Crisis Are We Talking About?. Int. J. Psychoanal, 83, 57-83. 


\section{HISTÓRIA DA PSICANÁLISE}

Garza-Guerrero, C. (2002b). Organisational and Educational Internal Impediments of Psychoanalysis: Contemporary Challenges. Int. J. Psychoanal, 83, 1407-1433.

Garza-Guerrero, C. (2004). Reorganisational and educational demands of psychoanalytic training today: Our long and marasmic night of one century. Int. J. Psychoanal, 85, 3-25.

Gay, P. (2006). Freud: A life for our time. New York, NY: Norton.

Gellner, E. (2003). The psychoanalytic movement: The cunning of unreason. Malden, MA: Blackwell

Grosskurth, P. (1991). Freud. L'anneau secret (C. Anthony, Trad.). Paris, France: P.U.F. Jones, E. (1957). Vida y obra de Sigmund Freud (Edición abreviada). Vol. III. Barcelona: Anagrama.

Kernberg, O. (2006a). The coming changes in psychoanalytic education: Part I. Int $J$ Psychoanal, 87: 1650-1673.

Kernberg, O. (2006b). Psychoanalytic Controversies: The pressing need to increase research in and on psychoanalysis. Int. J. Psychoanal, 87, 919-936.

Kernberg, O. (2007). The coming changes in psychoanalytic education: Part II. Int. J. Psychoanal, 88, 183-202.

Kernberg, O. (2011). Psychoanalysis and the university: A difficult relationship. Int. J. Psychoanal, 92, 609-622.

Laplanche, J. (2004). Pour la psychanalyse à l'Université. Recherches en psychanalyse; 1, 9-13.

Long, C., Eagle, G., \& Stevens, G. (2015).The clinician in the university: Reflections on a South African psychoanalytically oriented doctoral programme. Int. Psychoanal. doi:10.1111/1745-8315.12439.

Mészáros, J. (2012). Effect of Dictatorial Regimes on the Psychoanalytic Movement in Hungary before and after World War II. In J. Damousi, \& M. B. Plotkin (2012). Psychoanalysis and politics: Histories of psychoanalysis under conditions of restricted political freedom. Oxford: Oxford University Press.

Moreau, Ch. (1983). Freud y el ocultismo. Buenos Aires, Argentina: Gedisa.

Rodrigué, E. (1996). Sigmund Freud. El Siglo Del Psicoanálisis. Buenos Aires, Argentina: Sudamericana.

Roudinesco, E., \& Plon, M. (1997). Dictionnaire de la psychanalyse. Paris, France: Fayard.

Roussillon, R. (2010). Some comments on psychoanalysis in the French university system. International Forum of Psychoanalysis, 19(4), 201-203.

Touraine, A. (1995). Producción de la Sociedad. México: UNAM - IFAL.

Wallerstein, R. S. (2009). Psychoanalysis in the university: A full-time vision1. Int. J. Psychoanal, 90, 1107-1121. 
Wallerstein, R. S. (2011). Psychoanalysis in the university: The natural home for education and research. Int. J. Psychoanal, 92, 623-639.

\section{Resumenes}

(Freud e Ferenczi: algumas considerações sobre a criação da primeira cadeira de psicanálise na universidade)

O presente artigo expõe e analisa as reflexões que sustentaram Freud e Ferenczi durante o processo de criação da primeira cátedra de psicanálise na Universidade de Budapeste. A partir desses elementos, destaca-se que não foram apenas as diferenças teóricas ou profissionais que marcaram a relação conflitiva entre a psicanálise e a universidade, senão que nesse processo influiu, de maneira decisiva, a politica institucional que fundamentou a constituição do movimento psicanalítico.

Palavras-chave: Freud, Ferenczi, universidade, movimento psicanalítico

(Freud and Ferenczi: some thoughts on the creation of the first chair of psychoanalysis at the university)

This paper presents and analyzes reflections by Freud and Ferenczi as they were creating the first chair of psychoanalysis at the University of Budapest. Based on these elements, we emphasize that the conflictive relationship between psychoanalysis and the University was not only marked by theoretical or professional differences, but that this process was decisively influenced by the institutional policy that laid the foundations for the constitution of the psychoanalytic movement.

Key words: Freud, Ferenczi, university, psychoanalytic , movement

(Freud et Ferenczi: quelques considérations sur la création de la première chaire de psychanalyse à l'université)

Cet article présente et analyse des réflexions menées par Freud et Ferenczi à l'occasion de la création de la première chaire de psychanalyse à l'Université de Budapest. A partir de ces éléments, on souligne que le rapport conflictuel entre la psychanalyse et l'université n'a pas été uniquement marqué par de différences théoriques ou professionnelles, mais que ce processus a également été influencé de façon décisive par la politique institutionnelle qui a servi de base à la constitution du mouvement psychanalytique.

Mots clés: Freud, Ferenczi, université, mouvement psychanalytique 


\section{HISTÓRIA DA PSICANÁLISE}

(Freud und Ferenczi: Überlegungen zur Schaffung des ersten Lehrstuhls für Psychoanalyse an der Universität)

Dieser Artikel präsentiert und analysiert die Überlegungen Freuds und Ferenczis zur Schaffung des ersten Lehrstuhls für Psychoanalyse an der Universität Budapest. Aus diesen geht hervor, dass es nicht nur theoretische oder berufliche Meinungsverschiedenheiten waren, die die konfliktreiche Beziehung zwischen der Psychoanalyse und der Universität kennzeichneten. Der Prozess wurde ebenfalls entscheidend von der institutionellen Politik beeinflusst, die zur Verfassung der psychoanalytischen Bewegung beitrug.

Schlüsselwörter: Freud, Ferenczi, Universität, psychoanalytische Bewegung

Citação/Citation: Pizarro Obaid, F. (2018, junho). Freud y Ferenczi: algunas consideraciones sobre la creación de la primera cátedra de psicoanálisis en la Universidade. Revista Latinoamericana de Psicopatologia Fundamental, 21(2), 364-377. http://dx.doi.org/10.1590/ $1415-4714.2018 \mathrm{v} 21 \mathrm{n} 2 \mathrm{p} 364.9$

Editores do artigo/Editors: Profa. Dra. Ana Maria Rudge

Recebido/Received: 5.12.2017/ 12.5.2017 Aceito/Accepted: 18.3.2018 / 3.18.2018

Copyright: (C) 2009 Associação Universitária de Pesquisa em Psicopatologia Fundamental/ University Association for Research in Fundamental Psychopathology. Este é um artigo de livre acesso, que permite uso irrestrito, distribuição e reprodução em qualquer meio, desde que o autor e a fonte sejam citados / This is an open-access article, which permits unrestricted use, distribution, and reproduction in any medium, provided the original authors and sources are credited.

Financiamento/Funding: O autor declara não tere sido financiado ou apoiado / The author has no support or funding to report.

Conflito de interesses/Conflict of interest: $\mathrm{O}$ autor declara que não há conflito de interesses

/ The author has no conflict of interest to declare.

\section{Francisco Pizarro Obaid}

Licenciado en Psicología, Universidad Diego Portales (Chile); Magister en Psicología Clínica mención Psicoanálisis, Universidad Diego Portales (Chile); Doctor, Universidad Paris 7 - Dénis Diderot; École Doctorale Recherches en Psychanalyse et psychopathologie (Francia).

Facultad de Psicología, Universidad Diego Portales (Chile)

\# Grajales 1898; COD. POST. 8370076

Santiago, Chile

francisco.pizarro@udp.cl

This is an open-access article, which permits unrestricted use, distribution,

(cc) BY-NC and reproduction in any medium for non-commercial purposes provided the original authors and sources are credited. 